\title{
Normalisation of Hamiltonian in photogravitational elliptic restricted three body problem with Poynting-Robertson drag
}

\author{
Vivek Kumar Mishra*, Bhola Ishwar \\ Department of Mathematics, B.R.A.Bihar University,Muzaffarpur-842001, India \\ *Corresponding author E-mail:vivek.mishra2626@gmail.com
}

\begin{abstract}
In this paper, we have performed first order normalization in the photogravitational elliptic restricted three body problem with Poynting-Robertson drag. We suppose that bigger primary as radiating and smaller primary is an oblate spheroid. We have found the Lagrangian and the Hamiltonian of the problem. Then, we have expanded the Lagrangian function in power series of $\mathrm{x}$ and $\mathrm{y}$, where ( $\mathrm{x}$, y) are the coordinates of the triangular equilibrium points. Using Whittaker (1965) method, we have found that the second order part $\mathrm{H}_{2}$ of the Hamiltonian is transformed into the normal form $H_{2}=\omega_{1} I_{1}-\omega_{2} I_{2}$.
\end{abstract}

Keywords: First-Order Normalization/ERTBP/Photogravitational/P-R Drag.

\section{Introduction}

The elliptic restricted three body problem (ERTBP) is a generalization of the classical restricted three body problem (RTBP). It describes the three dimensional motion of a small particle, called the third body (infinitesimal mass) under the gravitational attraction force of two finite bodies, called the primaries, which revolve in elliptic orbit in a plane around their common centre of mass. The infinitesimal mass moves in the plane of motion of the primaries and does not influence the motion of the primaries.

Radiation, oblateness and a drag force known as PoyntingRobertson (P-R) drag also affects the motion of infinitesimal mass. Hence, many researchers studied the effect of these forces. Wyatt and Whipple (1950) have shown that P-R effect has been of very little significance. Chernikov (1970) has dealt with the SunPlanet-Particle model and concludes that due to P-R drag, triangular points are unstable. Schuerman (1980) studied the classical RTBP by including the radiation pressure and P-R effect. Murray (1994) investigated location and stability of the five Lagrangian points in the CRTBP when infinitesimal mass is acted by a variety of drag forces. Kushvah and Ishwar (2006) examined the linear stability of generalized photogravitational RTBP with P-R drag. We have already studied the linear stability of the given problem. We have found that in linear case, triangular equilibrium points are unstable. So our aim is to study the non-linear stability of triangular points. Liapunov's theorem (1956), Arnold's theorem (1961) and Moser's conditions (1962) played a significant role in deciding the non-linear stability of an equilibrium point. Moser (1962) gave some modification in Arnol's (1961) theorem. Then Deprit and Deprit-Bartholome (1962) investigated the non-linear stability of triangular points by applying Moser's modified version of Arnold's theorem (1961). Bhatnagar and Hallan (1983) studied the effect of perturbations on the non-linear stability of triangular points. Maciejewski and Gozdziewski (1991) described the normalization algorithms of Hamiltonian near an equilibrium point. Further, Bhatnagar, Gupta and Bhardwaj (1994) examined the non-linear stability of $\mathrm{L}_{4}$ for perturbed potential. Mishra and Ish war (1995) studied second order normalization in the generalized restricted problem of three bodies, smaller primary being an oblate spheroid. Ishwar (1997) studied non linear stability in the generalized restricted three body problem. J.Singh (2010) studied the combined effect of perturbations, radiation and oblateness on the non linear stability of triangular points in the restricted three body problem. Kushvah and Ishwar (2006) studied the second order normalization in the generalized photogravitational restricted three body problem with Poynting-Robertson drag.

The present study aims to perform first order normalization in the photogravitational elliptic restricted three body problem with P-R drag. We suppose that bigger primary is radiating and smaller an oblate spheroid. Using Whittaker (1965) method the second order part $\mathrm{H}_{2}$ of the Hamiltonian is transformed into the normal form $\mathrm{H}_{2}=\omega_{1} \mathrm{I}_{1}-\omega_{2} \mathrm{I}_{2}$. This paper is divided in four sections. Section 2 contains equations of motion and location of triangular equilibrium points. In section 3 , we have performed the first order normalization of $\mathrm{H}_{2}$, while section 4 concludes the result.

\section{Equations of motion and location of trian- gular equilibrium points}

We consider two bodies (primaries) of masses $m_{1}$ and $m_{2}$ with $\mathrm{m}_{1}>\mathrm{m}_{2}$ moving in a plane around their common center of mass in elliptic orbit and a third body (infinitesimal mass) of mass $m$ is moving in a plane of motion of the primaries. Equations of motion of our problem in rotating and pulsating co-ordinate system are given by (Sahoo and Ishwar 2000):

$\mathrm{x}^{\prime \prime}-2 \mathrm{y}^{\prime}=\frac{\partial \Omega}{\partial \mathrm{x}}-\frac{\mathrm{W}_{1} \mathrm{~N}_{1}}{\mathrm{n}^{2} \mathrm{r}_{1}^{2} \sqrt{1-\mathrm{e}^{2}}}=\mathrm{U}_{\mathrm{x}}$
$\mathrm{y}^{\prime \prime}+2 \mathrm{x}^{\prime}=\frac{\partial \Omega}{\partial \mathrm{y}}-\frac{\mathrm{W}_{1} \mathrm{~N}_{2}}{\mathrm{n}^{2} \mathrm{r}_{1}^{2} \sqrt{1-\mathrm{e}^{2}}}=\mathrm{U}_{\mathrm{y}}$,

where the force function 
$\mathrm{U}=\frac{1}{\sqrt{1-\mathrm{e}^{2}}}\left[\frac{\mathrm{x}^{2}+\mathrm{y}^{2}}{2}+\frac{1}{\mathrm{n}^{2}}\left\{\frac{(1-\mu) \mathrm{q}_{1}}{\mathrm{r}_{1}}+\frac{\mu}{\mathrm{r}_{2}}+\frac{\mu \mathrm{A}_{2}}{2 \mathrm{r}_{2}^{3}}+\mathrm{W}_{1}\left(\frac{(\mathrm{x}+\mu) \mathrm{x}^{\prime}+\mathrm{yy}^{\prime}}{2 \mathrm{r}_{1}^{2}}-\right.\right.\right.$ $\left.\left.\left.\mathrm{n} \arctan \left(\frac{\mathrm{y}}{\mathrm{x}+\mu}\right)\right)\right\}\right]$

$\Omega=\frac{1}{\sqrt{1-\mathrm{e}^{2}}}\left[\frac{\mathrm{x}^{2}+\mathrm{y}^{2}}{2}+\frac{1}{\mathrm{n}^{2}}\left\{\frac{(1-\mu) \mathrm{q}_{1}}{\mathrm{r}_{1}}+\frac{\mu}{\mathrm{r}_{2}}+\frac{\mu \mathrm{A}_{2}}{2 \mathrm{r}_{2}^{3}}\right\}\right]$

$\mathrm{N}_{1}=\frac{(\mathrm{x}+\mu) \mathrm{N}}{\mathrm{r}_{1}^{2}}+\mathrm{x}^{\prime}-\mathrm{ny}$

$\mathrm{N}_{2}=\frac{\mathrm{yN}}{\mathrm{r}_{1}^{2}}+\mathrm{y}^{\prime}+\mathrm{n}(\mathrm{x}+\mu)$

$N=(x+\mu) x^{\prime}+y y^{\prime}$

$\mathrm{W}_{1}=\frac{(1-\mu)\left(1-\mathrm{q}_{1}\right)}{\mathrm{c}_{\mathrm{d}}}$.

Here, dash (') represents differentiation with respect to eccentric anomaly (E). The mean motion of our problem is given by:

$\mathrm{n}^{2}=\frac{1}{\mathrm{a}}\left(1+\frac{3 \mathrm{e}^{2}}{2}+\frac{3 \mathrm{~A}_{2}}{2}\right)$

$r_{i}=\left(x+x_{i}\right)^{2}+y^{2}+z^{2}(i=1,2)$

$\mathrm{x}_{1}=-\mu, \mathrm{x}_{2}=1-\mu, \mu=\frac{\mathrm{m}_{2}}{\mathrm{~m}_{1}+\mathrm{m}_{2}}$

Here, $m_{1}, m_{2}$ are the masses of the bigger and smaller primaries. $\left(x_{1}, 0,0\right)$ and $\left(x_{2}, 0,0\right)$ are the coordinate of $m_{1}$ and $m_{2}$ respectively. $\mathrm{q}_{1}$ is mass reduction factor and $\mathrm{W}_{1}$ is $\mathrm{P}-\mathrm{R}$ drag due to bigger primary $m_{1} \cdot A_{2}=\frac{r_{e}^{2}-r_{p}^{2}}{5 r^{2}}$ is oblateness coefficient due to smaller primary $\mathrm{m}_{2}$, where $\mathrm{r}_{\mathrm{e}}, \mathrm{r}_{\mathrm{p}}$ represents equatorial radii and polar radii respectively. $r_{i}(i=1,2)$ are the distances of the infinitesimal mass from $m_{1}$ and $m_{2}$ respectively. Semi-major axis and eccentricity of orbit is denoted by a and e respectively. $\mathrm{c}_{\mathrm{d}}$ is dimensionless velocity of light.

Using perturbation method, we have found location of triangular equilibrium point. For triangular equilibrium points $\mathrm{U}_{\mathrm{x}}=0, \mathrm{U}_{\mathrm{y}}=$ $0, y \neq 0$ and $z=0$ then we have

$\mathrm{x}=\frac{1}{2}-\mu+\frac{1}{2}\left[\left(\mathrm{aq}_{1}\right)^{2 / 3}\left(1-\mathrm{A}_{2}-\mathrm{e}^{2}\right)-\mathrm{a}^{2 / 3}\left(1-\mathrm{A}_{2}-\mathrm{e}^{2}+\right.\right.$

$\left.\mathrm{A}_{2} \mathrm{a}^{-2 / 3}\right]+\frac{\mathrm{W}_{1} \mathrm{a}^{1 / 2}}{3 \mathrm{y}_{0}(1-\mu) \mu}\left\{\left(1+\frac{\mathrm{A}_{2}}{4}-\frac{3 \mathrm{e}^{2}}{4}\right) \frac{\mu}{2}-\left(\mathrm{a}^{2 / 3}+\left(\mathrm{aq}_{1}\right)^{2 / 3}\right)(1+\right.$

$\left.\left.\frac{\mathrm{A}_{2}}{4}-\frac{7 \mathrm{e}^{2}}{4}\right) \frac{\mu}{2}-(1-\mu) \mathrm{a}^{2 / 3}\left(1+\frac{\mathrm{A}_{2}}{4}-2 \mathrm{~A}_{2} \mathrm{a}^{-2 / 3}-\frac{7 \mathrm{e}^{2}}{4}\right)\right\}$

$\mathrm{y}= \pm\left[\left(\mathrm{aq}_{1}\right)^{2 / 3}\left(1-\mathrm{A}_{2}-\mathrm{e}^{2}\right)-\frac{1}{4}\left\{1+2\left(\left(\mathrm{aq}_{1}\right)^{2 / 3}-\mathrm{a}^{2 / 3}\right)(1-\right.\right.$

$\left.\mathrm{e}^{2}\right)+\left(\left(\mathrm{aq}_{1}\right)^{2 / 3}-\mathrm{a}^{2 / 3}\right)^{2}\left(1-2 \mathrm{e}^{2}\right)-2 \mathrm{~A}_{2}\left(1+\left(\mathrm{aq}_{1}\right)^{2 / 3}-\right.$

$\left.\left.\mathrm{a}^{2 / 3}\right)^{2}\right\}+\frac{\mathrm{w}_{1} \mathrm{a}^{1 / 2}}{3 \mathrm{y}_{0}(1-\mu) \mu}\left\{\left(1+\frac{\mathrm{A}_{2}}{4}-\frac{3 \mathrm{e}^{2}}{4}\right) \frac{\mu}{2}-\mu\left(\mathrm{aq}_{1}\right)^{2 / 3}\left(1+\frac{\mathrm{A}_{2}}{4}-\right.\right.$

$\left.\frac{7 \mathrm{e}^{2}}{4}\right)+\left(\left(\mathrm{aq}_{1}\right)^{4 / 3}-\mathrm{a}^{4 / 3}\right)\left(1+\frac{\mathrm{A}_{2}}{4}-\frac{11 \mathrm{e}^{2}}{4}\right) \frac{\mu}{2}+(1-\mu) \mathrm{a}^{2 / 3}(1+$

$\left.\frac{\mathrm{A}_{2}}{4}-2 \mathrm{~A}_{2} \mathrm{a}^{-2 / 3}-\frac{7 \mathrm{e}^{2}}{4}\right)+(1-\mu) \mathrm{a}^{2 / 3}\left(\left(\mathrm{aq}_{1}\right)^{2 / 3}-\mathrm{a}^{2 / 3}\right)\left(1+\frac{\mathrm{A}_{2}}{4}-\right.$

$\left.2 \mathrm{~A}_{2} \mathrm{a}^{-2 / 3}-\frac{11 \mathrm{e}^{2}}{4}\right)-\frac{\mu}{2} \mathrm{~A}_{2}\left(1+\left(\mathrm{aq}_{1}\right)^{2 / 3}-\mathrm{a}^{2 / 3}\right)\left(\left(\mathrm{aq}_{1}\right)^{2 / 3}+\right.$

$\left.\left.\left.a^{2 / 3}-1\right)-(1-\mu) a^{2 / 3}\left(1+\left(a q_{1}\right)^{2 / 3}-a^{2 / 3}\right) A_{2}\right\}\right]^{1 / 2}$

where $y_{0}= \pm\left[\delta^{2}\left(1-\mathrm{e}^{2}\right)-\frac{1}{4}\left\{1+2\left(\delta^{2}-\mathrm{a}^{2 / 3}\right)\left(1-\mathrm{e}^{2}\right)\right\}\right]$,

$$
\delta=\left(\mathrm{aq}_{1}\right)^{1 / 3}
$$

\section{First order normalization}

The Lagrangian function of our problem is written as:
$\mathrm{L}=\frac{1}{2}\left(\dot{\mathrm{x}}^{2}+\dot{\mathrm{y}}^{2}\right)+\frac{\dot{\mathrm{v}}^{2}}{2}\left(\mathrm{x}^{2}+\mathrm{y}^{2}\right)+\dot{\mathrm{v}}(\mathrm{x} \dot{\mathrm{y}}-\dot{\mathrm{x}} \mathrm{y})+\frac{(1-\mu) \mathrm{q}_{1}}{\mathrm{r}_{1}}+\frac{\mu}{\mathrm{r}_{2}}+$

$\frac{\mu A_{2}}{2 r_{2}^{3}}+W_{1}\left(\frac{(x+\mu) \dot{x}+y \dot{y}+z \dot{z}}{2 r_{1}^{2}}-n \arctan \left(\frac{y}{x+\mu}\right)\right)$

where $\mathrm{v}$ is true anomaly and the Hamiltonian $\mathrm{H}=-\mathrm{L}+\mathrm{p}_{\mathrm{x}} \dot{\mathrm{x}}+$ $\mathrm{p}_{\mathrm{y}} \dot{\mathrm{y}}, \mathrm{p}_{\mathrm{x}}, \mathrm{p}_{\mathrm{y}}$ are the momenta coordinates given by $\mathrm{p}_{\mathrm{x}}=\frac{\partial \mathrm{L}}{\partial \dot{\mathrm{x}}}=\dot{\mathrm{x}}-$ $\dot{\mathrm{v} y}+\frac{\mathrm{W}_{1}(\mathrm{x}+\mu)}{2 \mathrm{r}_{1}^{2}}, \mathrm{p}_{\mathrm{y}}=\frac{\partial \mathrm{L}}{\partial \dot{y}}=\dot{\mathrm{y}}-\dot{\mathrm{v} x}+\frac{\mathrm{W}_{1} \mathrm{y}}{2 \mathrm{r}_{1}^{2}}$. For simplicity, we suppose $\mathrm{q}_{1}=1-\epsilon$ with $\epsilon \ll 1$. Then triangular equilibrium points takes the form

$\mathrm{x}=\frac{1}{2}-\mu-\frac{\mathrm{a}^{2 / 3} \epsilon}{3}+\frac{\mathrm{A}_{2} \mathrm{a}^{2 / 3} \in}{3}+\frac{\mathrm{a}^{2 / 3} \in \mathrm{e}^{2}}{3}-\frac{\mathrm{A}_{2}}{2}+\frac{2 \sqrt{3} \mathrm{~W}_{1} \mathrm{a}^{1 / 2}}{9 \mu(1-\mu)}\left[\frac{\mu}{6}(1-\right.$

$\left.\frac{3 \mathrm{e}^{2}}{4}+\frac{\mathrm{A}_{2}}{4}\right)+\left(1-\frac{7 \mathrm{e}^{2}}{4}+\frac{\mathrm{A}_{2}}{4}\right)\left(\frac{\mu \in \mathrm{a}^{2 / 3}}{36}-\frac{\mathrm{a}^{2 / 3}}{3}\right)-\frac{\mathrm{a}^{2 / 3}}{3}\left(1-\frac{11 \mathrm{e}^{2}}{4}-\right.$

$\left.\frac{3 \mathrm{~A}_{2}}{4}\right)+\left(1-\frac{7 \mathrm{e}^{2}}{4}-\frac{3 \mathrm{~A}_{2}}{4}\right)\left(\frac{\mu \epsilon}{6}-\frac{\mathrm{a}^{2 / 3 \mu}}{4}\right)+2 \mathrm{~A}_{2}(1-\mu)\left(\frac{1}{3}-\frac{\mathrm{a}^{2 / 3}}{2}-\frac{\epsilon}{3}+\right.$

$\left.\left.\frac{a^{2 / 3 \epsilon}}{6}\right)\right]$

$\mathrm{y}=\frac{\sqrt{3}}{2}\left[1+\frac{\mathrm{a}^{2 / 3}}{2}\left(1-\mathrm{e}^{2}\right)\left(1-\frac{\epsilon}{3}\right)-\frac{\mathrm{A}_{2}}{2}\left(1+\mathrm{a}^{2 / 3}\right)+\frac{4}{3}\left(\mathrm{~A}_{2} \mathrm{a}^{2 / 3} \in\right.\right.$ $-1)-\frac{A_{2}}{2}+\frac{4 W_{1} a^{1 / 2}}{9 \sqrt{3} \mu(1-\mu)}\left\{\frac{\mu}{6}\left(1-\frac{3 e^{2}}{4}+\frac{A_{2}}{4}\right)-\frac{\mu a^{2 / 3}}{3}\left(1-\frac{7 e^{2}}{4}+\frac{A_{2}}{4}\right)+\right.$ $\frac{2 \mathrm{\epsilon a}^{2 / 3}}{9}\left(1-\frac{7 \mathrm{e}^{2}}{4}+\frac{\mathrm{A}_{2}}{4}\right)+(1-\mu) \frac{\mathrm{a}^{2 / 3}}{3}\left(1-\frac{7 \mathrm{e}^{2}}{4}+\frac{\mathrm{A}_{2}}{4}-2 \mathrm{~A}_{2} \mathrm{a}^{-2 / 3}\right)+$ $\frac{\mu \mathrm{A}_{2}}{6}\left(1+\mathrm{a}^{2 / 3}-\frac{2 \epsilon \mathrm{a}^{2 / 3}}{3}+\frac{2 \epsilon}{3}\right)-\frac{\mathrm{A}_{2} \mathrm{a}^{2 / 3}(1-\mu)}{3}-\frac{\mu \mathrm{a}^{2 / 3}}{4}\left(1-\frac{7 \mathrm{e}^{2}}{4}-\frac{3 \mathrm{~A}_{2}}{4}\right)-$ $\frac{\mu \mathrm{A}_{2} \mathrm{a}^{2 / 3}}{4}\left(1+\frac{2 \epsilon}{3}\right)+\frac{\mu \epsilon}{6}\left(1-\frac{7 \mathrm{e}^{2}}{4}-\frac{3 \mathrm{~A}_{2}}{4}\right)-\frac{\mu \epsilon \mathrm{a}^{2 / 3}}{3}\left(1-\frac{11 \mathrm{e}^{2}}{4}-\frac{3 \mathrm{~A}_{2}}{4}\right)+$ $\frac{\epsilon \mathrm{a}^{2 / 3}(1-\mu)}{3}\left(1-\frac{7 \mathrm{e}^{2}}{4}+\frac{\mathrm{A}_{2}}{4}-2 \mathrm{~A}_{2} \mathrm{a}^{-2 / 3}\right)+\frac{\mu € \mathrm{~A}_{2}}{6}\left(1-\mathrm{e}^{2}-\mathrm{A}_{2}\right)(1+$ $\left.\mathrm{a}^{2 / 3}-\frac{2 \mathrm{\epsilon a}^{2 / 3}}{3}+\frac{2 \epsilon}{3}\right)-\frac{\mathrm{A}_{2} \mathrm{\epsilon a}^{2 / 3}}{3}(1-\mu)\left(1-\mathrm{e}^{2}-\mathrm{A}_{2}\right)+\frac{\mu \epsilon \mathrm{a}^{2 / 3}}{12}(1-$ $\left.\left.\left.\frac{7 \mathrm{e}^{2}}{4}+\frac{\mathrm{A}_{2}}{4}\right)+\frac{\mu \mathrm{A}_{2} \mathrm{ea}^{2 / 3}}{12}\right\}\right]$.

We shift the origin to $\mathrm{L}_{4}$ (triangular equilibrium point). For that, we change $\mathrm{x} \rightarrow \mathrm{x}_{*}+\mathrm{x}$ and $\mathrm{y} \rightarrow \mathrm{y}_{*}+\mathrm{y}$. We suppose $\mathrm{a}_{*}=\mathrm{x}_{*}+$ $\mu$ and $b_{*}=y_{*}$, so that

$\mathrm{a}_{*}=\frac{1}{2}-\frac{\mathrm{a}^{2 / 3} \in}{3}+\frac{\mathrm{A}_{2} \mathrm{a}^{2 / 3} \in}{3}+\frac{\mathrm{a}^{2 / 3} \in \mathrm{e}^{2}}{3}-\frac{\mathrm{A}_{2}}{2}+\frac{2 \sqrt{3} \mathrm{~W}_{1} \mathrm{a}^{1 / 2}}{9 \mu(1-\mu)}\left[\frac{\mu}{6}\left(1-\frac{3 \mathrm{e}^{2}}{4}+\right.\right.$

$\left.\frac{\mathrm{A}_{2}}{4}\right)+\left(1-\frac{7 \mathrm{e}^{2}}{4}+\frac{\mathrm{A}_{2}}{4}\right)\left(\frac{\mu \in \mathrm{a}^{2 / 3}}{36}-\frac{\mathrm{a}^{2 / 3}}{3}\right)-\frac{\mathrm{a}^{2 / 3}}{3}\left(1-\frac{11 \mathrm{e}^{2}}{4}-\frac{3 \mathrm{~A}_{2}}{4}\right)+$

$\left.\left(1-\frac{7 \mathrm{e}^{2}}{4}-\frac{3 \mathrm{~A}_{2}}{4}\right)\left(\frac{\mu \epsilon}{6}-\frac{\mathrm{a}^{2 / 3 \mu}}{4}\right)+2 \mathrm{~A}_{2}(1-\mu)\left(\frac{1}{3}-\frac{\mathrm{a}^{2 / 3}}{2}-\frac{\epsilon}{3}+\frac{\mathrm{a}^{2 / 3} \epsilon}{6}\right)\right]$

$\mathrm{b}_{*}=\frac{\sqrt{3}}{2}\left[1+\frac{\mathrm{a}^{2 / 3}}{2}\left(1-\mathrm{e}^{2}\right)\left(1-\frac{\epsilon}{3}\right)-\frac{\mathrm{A}_{2}}{2}\left(1+\mathrm{a}^{2 / 3}\right)+\right.$

$\frac{4}{3}\left(\mathrm{~A}_{2} \mathrm{a}^{2 / 3} \in-1\right)-\frac{\mathrm{A}_{2}}{2}+\frac{4 \mathrm{~W}_{1} \mathrm{a}^{1 / 2}}{9 \sqrt{3} \mu(1-\mu)}\left\{\frac{\mu}{6}\left(1-\frac{3 \mathrm{e}^{2}}{4}+\frac{\mathrm{A}_{2}}{4}\right)-\right.$ $\frac{\mu \mathrm{a}^{2 / 3}}{3}\left(1-\frac{7 \mathrm{e}^{2}}{4}+\frac{\mathrm{A}_{2}}{4}\right)+\frac{2 \mathrm{ea}^{2 / 3}}{9}\left(1-\frac{7 \mathrm{e}^{2}}{4}+\frac{\mathrm{A}_{2}}{4}\right)+(1-\mu) \frac{\mathrm{a}^{2 / 3}}{3}(1-$ $\left.\frac{7 \mathrm{e}^{2}}{4}+\frac{\mathrm{A}_{2}}{4}-2 \mathrm{~A}_{2} \mathrm{a}^{-2 / 3}\right)+\frac{\mu \mathrm{A}_{2}}{6}\left(1+\mathrm{a}^{2 / 3}-\frac{2 \epsilon \mathrm{a}^{2 / 3}}{3}+\frac{2 \epsilon}{3}\right)-$ $\frac{\mathrm{A}_{2} \mathrm{a}^{2 / 3}(1-\mu)}{3}-\frac{\mu \mathrm{a}^{2 / 3}}{4}\left(1-\frac{7 \mathrm{e}^{2}}{4}-\frac{3 \mathrm{~A}_{2}}{4}\right)-\frac{\mu \mathrm{A}_{2} \mathrm{a}^{2 / 3}}{4}\left(1+\frac{2 \mathrm{\epsilon}}{3}\right)+$ $\frac{\mu \epsilon}{6}\left(1-\frac{7 \mathrm{e}^{2}}{4}-\frac{3 \mathrm{~A}_{2}}{4}\right)-\frac{\mu \mathrm{\epsilon} \mathrm{a}^{2 / 3}}{3}\left(1-\frac{11 \mathrm{e}^{2}}{4}-\frac{3 \mathrm{~A}_{2}}{4}\right)+\frac{\mathrm{\epsilon a}^{2} / 3(1-\mu)}{3}\left(1-\frac{7 \mathrm{e}^{2}}{4}+\right.$ $\left.\frac{\mathrm{A}_{2}}{4}-2 \mathrm{~A}_{2} \mathrm{a}^{-2 / 3}\right)+\frac{\mu \epsilon \mathrm{A}_{2}}{6}\left(1-\mathrm{e}^{2}-\mathrm{A}_{2}\right)\left(1+\mathrm{a}^{2 / 3}-\frac{2 \epsilon \mathrm{a}^{2 / 3}}{3}+\frac{2 \epsilon}{3}\right)-$ $\left.\left.\frac{\mathrm{A}_{2} \mathrm{\epsilon a}^{2 / 3}}{3}(1-\mu)\left(1-\mathrm{e}^{2}-\mathrm{A}_{2}\right)+\frac{\mu \mathrm{ea}^{2 / 3}}{12}\left(1-\frac{7 \mathrm{e}^{2}}{4}+\frac{\mathrm{A}_{2}}{4}\right)+\frac{\mu \mathrm{A}_{2} \mathrm{\epsilon a}^{2 / 3}}{12}\right\}\right]$. Expanding $\mathrm{L}$ in power series of $\mathrm{x}$ and $\mathrm{y}$, we get

$$
\mathrm{L}=\mathrm{L}_{0}+\mathrm{L}_{1}+\mathrm{L}_{2}+\mathrm{L}_{3}---------
$$

$\mathrm{H}=\mathrm{H}_{0}+\mathrm{H}_{1}+\mathrm{H}_{2}+\mathrm{H}_{3}----=-\mathrm{L}+\mathrm{p}_{\mathrm{x}} \dot{\mathrm{x}}+\mathrm{p}_{\mathrm{y}} \dot{\mathrm{y}}$ where $L_{0}, L_{1}, L_{2}, L_{3}$ are constant, first order term, second order term respectively. Second order term $\mathrm{H}_{2}$ of Hamiltonian is written as:

$\mathrm{H}_{2}=\frac{\mathrm{p}_{\mathrm{x}}^{2}+\mathrm{p}_{\mathrm{y}}^{2}}{2}+\dot{\mathrm{v}}\left(\mathrm{yp} \mathrm{p}_{\mathrm{x}}-\mathrm{xp}_{\mathrm{y}}\right)+\mathrm{Ex}^{2}+\mathrm{Gxy}+\mathrm{Fy}^{2}$ 
$\mathrm{E}=\frac{\mathrm{a}^{2 / 3}}{16}\left[-1+3 \mathrm{a}^{2 / 3}-3 \epsilon-3 \epsilon \mathrm{a}^{2 / 3}-2 \mathrm{~A}_{2}-\mathrm{A}_{2} \mathrm{a}^{2 / 3}-\right.$

$9 \mathrm{~A}_{2} \epsilon \mathrm{a}^{2 / 3}-\frac{13 \mathrm{~A}_{2} \epsilon}{2}-\frac{5}{4}(1+\mu) \mathrm{e}^{2} \mathrm{a}^{2 / 3}+\frac{\mathrm{e}^{2} \epsilon}{4}-\frac{\mathrm{W}_{1} \mathrm{a}^{2 / 3}}{3 \sqrt{3}}\left(1+\frac{3 \mathrm{e}^{2}}{4}+\right.$

$\left.\frac{3 \mathrm{~A}_{2}}{4}\right)\left\{\frac{17}{2}+\frac{\gamma}{2}-\frac{207 \epsilon}{9}-\frac{23 \gamma \epsilon}{3}+\frac{\mathrm{a}^{2} / 3}{3}\left(24+\frac{3 \gamma}{2}-\frac{211 \epsilon}{3}-22 \gamma \epsilon\right)+\right.$

$\left.\frac{\mathrm{a}^{4 / 3}}{3}\left(54-\frac{3 \gamma}{2}-\frac{196 \epsilon}{3}-5 \gamma \epsilon\right)\right\}+\gamma\left\{2 \epsilon \mathrm{a}^{2 / 3}-10 \mathrm{~A}_{2}+22 \mathrm{~A}_{2} \mathrm{a}^{2 / 3}+\right.$

$\frac{\mathrm{A}_{2} \mathrm{\epsilon a}^{2 / 3}}{3}+\frac{\mathrm{W}_{1} \mathrm{a}^{2 / 3}}{3 \sqrt{3}}\left(1+\frac{3 \mathrm{e}^{2}}{4}+\frac{3 \mathrm{~A}_{2}}{4}\right)\left\{54+\frac{9 \gamma}{2}-\frac{197 \epsilon}{9}-7 \gamma \epsilon+\right.$

$\left.\left.\left.\frac{\mathrm{a}^{2 / 3}}{3}\left(\frac{201}{2}+12 \gamma-\frac{203 \epsilon}{3}-\frac{100 \gamma \epsilon}{3}\right)+\frac{\mathrm{a}^{4 / 3}}{3}\left(36-\frac{52 \epsilon}{3}-\frac{35 \gamma \epsilon}{3}\right)\right\}\right\}\right]$

$\mathrm{F}=-\frac{\mathrm{a}^{2 / 3}}{16}\left[4+6 \mathrm{a}^{2 / 3}-4 \epsilon+2 \epsilon \mathrm{a}^{2 / 3}+15 \mathrm{~A}_{2}+6 \mathrm{~A}_{2} \mathrm{a}^{2 / 3}-\right.$

$39 \mathrm{~A}_{2} \mathrm{\epsilon a}^{2 / 3}-\frac{5}{4}(1+2 \mu) \mathrm{e}^{2} \mathrm{a}^{2 / 3}+\frac{\mathrm{e}^{2} \mathrm{\epsilon a}^{2 / 3}}{4}-\frac{\mathrm{W}_{1} \mathrm{a}^{2 / 3}}{3 \sqrt{3}}\left(1+\frac{3 \mathrm{e}^{2}}{4}+\right.$

$\left.\frac{3 \mathrm{~A}_{2}}{4}\right)\left\{\frac{37}{2}+\frac{11 \gamma}{2}-\frac{347 \epsilon}{9}+\frac{13 \gamma \epsilon}{3}+\frac{\mathrm{a}^{2} / 3}{3}\left(57+\frac{15 \gamma}{2}-\frac{278 \epsilon}{3}+8 \gamma \epsilon\right)+\right.$

$\left.\frac{\mathrm{a}^{4 / 3}}{3}\left(\frac{33}{2}+\frac{9 \gamma}{2}-\frac{201 \epsilon}{3}+5 \gamma \epsilon\right)\right\}+\gamma\left\{3 \epsilon+3 \epsilon \mathrm{a}^{2 / 3}-16 \mathrm{~A}_{2} \epsilon-\right.$

$\frac{5 \mathrm{~A}_{2} \mathrm{\epsilon a}^{2} / 3}{18}+\frac{\mathrm{W}_{1} \mathrm{a}^{1 / 2}}{3 \sqrt{3}}\left(1+\frac{3 \mathrm{e}^{2}}{4}+\frac{3 \mathrm{~A}_{2}}{4}\right)\left\{\frac{95}{2}+\frac{15 \gamma}{2}-\frac{514 \epsilon}{9}-\frac{\gamma \epsilon}{3}+\right.$

$\left.\left.\left.\frac{\mathrm{a}^{2 / 3}}{3}\left(\frac{243}{2}+\frac{25 \gamma}{2}-\frac{412 \epsilon}{3}-2 \gamma \epsilon\right)+\frac{\mathrm{a}^{4 / 3}}{3}\left(\frac{33}{2}-\frac{62 \epsilon}{3}+\frac{11 \gamma}{2}-\gamma \epsilon\right)\right\}\right\}\right]$

$\mathrm{G}=\frac{\sqrt{3} \mathrm{a}^{2 / 3}}{8}\left[3 \epsilon-\epsilon \mathrm{a}^{2 / 3}+5 \mathrm{~A}_{2}+\mathrm{A}_{2} \mathrm{a}^{2 / 3}-\frac{15 \mathrm{~A}_{2} \epsilon}{2}-11 \mathrm{~A}_{2} \epsilon \mathrm{a}^{2 / 3}-\right.$

$\frac{3}{4}(1+\mu) \mathrm{e}^{2} \mathrm{a}^{2 / 3}+\frac{\mathrm{e}^{2} \mathrm{ea}^{2 / 3}}{4}-\frac{\mathrm{W}_{1} \mathrm{a}^{2 / 3}}{3 \sqrt{3}}\left(1+\frac{3 \mathrm{e}^{2}}{4}+\frac{3 \mathrm{~A}_{2}}{4}\right)\left\{\frac{9}{2}+\frac{5 \gamma}{6}-\frac{88 \epsilon}{9}+\right.$

$\left.\frac{8 \gamma \epsilon}{9}+\frac{\mathrm{a}^{2} / 3}{3}\left(33+\frac{2 \gamma}{2}-\frac{40 \epsilon}{3}+\frac{5 \gamma \epsilon}{3}\right)+\frac{\mathrm{a}^{4 / 3}}{3}\left(12+\gamma-10 \epsilon+\frac{\gamma \epsilon}{3}\right)\right\}+$

$\gamma\left\{\frac{15 \mathrm{~A}_{2} \epsilon}{2}+9 \mathrm{~A}_{2} \mathrm{\epsilon a}^{2 / 3}-13 \mathrm{~A}_{2}-\frac{11 \epsilon}{3}-2 \mathrm{\epsilon a}^{2 / 3}+\frac{\mathrm{W}_{1} \mathrm{a}^{1 / 2}}{3 \sqrt{3}}\left(1+\frac{3 \mathrm{e}^{2}}{4}+\right.\right.$

$\left.\frac{3 \mathrm{~A}_{2}}{4}\right)\left\{-\frac{15}{2}+\frac{3 \gamma}{6}-\frac{98 \epsilon}{3}+\frac{2 \gamma \epsilon}{9}+\frac{\mathrm{a}^{2 / 3}}{3}\left(-18+\frac{5 \gamma}{2}+45 \epsilon+\frac{2 \gamma \epsilon}{9}\right)+\right.$

$\left.\left.\left.\frac{\mathrm{a}^{4 / 3}}{3}\left(-9+43 \epsilon+\frac{\gamma \epsilon}{9}+\frac{\gamma}{2}\right)\right\}\right\}\right]$

and $\gamma=1-2 \mu$.

Now, we perform transformation from the phase space $\left(x, y, p_{x} p_{y}\right)$ into the phase space $\left(\emptyset_{1}, \emptyset_{2}, I_{1}, I_{2}\right)$, with the help of Whittaker (1965) method. We suppose the following set of linear equations of variables $\mathrm{x}$ and $\mathrm{y}$

$$
\begin{array}{ll}
-\lambda \mathrm{p}_{\mathrm{x}}=\frac{\partial \mathrm{H}_{2}}{\partial \mathrm{x}} & \lambda_{\mathrm{x}}=\frac{\partial \mathrm{H}_{2}}{\partial \mathrm{p}_{\mathrm{x}}} \\
-\lambda \mathrm{p}_{\mathrm{y}}=\frac{\partial \mathrm{H}_{2}}{\partial \mathrm{y}} & \lambda_{\mathrm{y}}=\frac{\partial \mathrm{H}_{2}}{\partial \mathrm{p}_{\mathrm{y}}}
\end{array}
$$

that is $\mathrm{AX}=0$

$$
X=\left[\begin{array}{l}
x \\
y \\
p_{x} \\
p_{y}
\end{array}\right], A=\left[\begin{array}{cccc}
2 E & G & \lambda & -n \\
G & 2 F & n & \lambda \\
-\lambda & n & 1 & 0 \\
-n & -\lambda & 0 & 1
\end{array}\right]
$$

Clearly $|\mathrm{A}|=0$ implies that the characterstic equation corresponding to Hamiltonian is given by

$\lambda^{4}+2\left(E+F+n^{2}\right) \lambda^{2}+4 E F-G^{2}+n^{4}-2 n^{2}(E+F)=0$.

Discriminant is given by

$D=4\left(E+F+n^{2}\right)^{2}-4\left\{4 E F-G^{2}+n^{4}-2 n^{2}(E+F)\right\}$.

Stability is assured only when $\mathrm{D}>0$. WhenD $>0$

the roots $\pm i \omega_{1}$ and $\pm i \omega_{2}$ are related to each other as

$\omega_{1}^{2}+\omega_{2}^{2}=\frac{a^{2 / 3}}{8}\left[-5-3 a^{2 / 3}+\epsilon-5 \epsilon a^{2 / 3}-17 A_{2}-7 A_{2} a^{2 / 3}+\right.$ $\frac{127 A_{2} \epsilon a^{2 / 3}}{6}+\frac{5}{4} \mu e^{2} a^{2 / 3}+\frac{e^{2} \epsilon}{4}\left(1-a^{2 / 3}\right)-\frac{W_{1} a^{2 / 3}}{3 \sqrt{3}}\left(1+\frac{3 e^{2}}{4}+\right.$ $\left.\frac{3 A_{2}}{4}\right)\left\{-10-5 \gamma-\frac{140 \epsilon}{9}-12 \gamma \epsilon+\frac{a^{2 / 3}}{3}\left(-33-6 \gamma-\frac{67 \epsilon}{3}-\right.\right.$

$\left.30 \gamma \epsilon)+\frac{a^{4 / 3}}{3}\left(66-3 \gamma-\frac{5 \epsilon}{3}-10 \gamma \epsilon\right)\right\}+\gamma\left\{-\epsilon a^{2 / 3}-10 A_{2}-\right.$

$3 \epsilon+16 A_{2} \epsilon+22 A_{2} a^{2 / 3}+\frac{11 A_{2} \epsilon a^{2 / 3}}{18}+\frac{W_{1} a^{2 / 3}}{3 \sqrt{3}}\left(1+\frac{3 e^{2}}{4}+\right.$

$\left.\frac{3 A_{2}}{4}\right)\left\{3-3 \gamma+\frac{514 \epsilon}{9}-\frac{20 \gamma \epsilon}{3}+\frac{a^{2 / 3}}{3}\left(-21-\frac{\gamma}{2}+\frac{168 \epsilon}{3}-\frac{94 \gamma \epsilon}{3}\right)+\right.$

$\left.\left.\left.\frac{a^{4 / 3}}{3}\left(\frac{39}{2}+\frac{10 \epsilon}{3}-\frac{11 \gamma}{2}-\frac{32 \gamma \epsilon}{3}\right)\right\}\right\}\right]+\frac{2}{a}\left(1+\frac{3 e^{2}}{2}+\frac{3 A_{2}}{2}\right)$

$\omega_{1}^{2} \omega_{2}^{2}=\frac{19 a^{2 / 3}}{16}-\frac{1}{2}-\frac{5 \gamma^{2}}{8}-\frac{17 \gamma^{2} a^{2 / 3}}{16}+\frac{9 \epsilon}{8}+\frac{9 \gamma \epsilon a^{2 / 3}}{8}-\frac{\gamma^{2} \epsilon}{4}-$ $\frac{\gamma^{2} \epsilon a^{2 / 3}}{8}+\frac{97 \gamma A_{2}}{16}+\frac{5 \gamma A_{2} a^{2 / 3}}{4}-3 \gamma A_{2} a^{2 / 3}-\frac{177 A_{2} \epsilon}{32}-2 A_{2} \epsilon a^{2 / 3}+$ $\frac{2485 \gamma \epsilon A_{2} a^{2 / 3}}{192}-\frac{5000 \gamma \epsilon A_{2}}{192}+\frac{25 e^{2} \gamma \epsilon}{16}+\frac{7 e^{2} \gamma \epsilon a^{2 / 3}}{16}-\frac{34 e^{2} \gamma^{2} \epsilon}{16}+$ $\frac{W_{1} a^{2 / 3}}{3 \sqrt{3}}\left(1+\frac{3 e^{2}}{4}+\frac{3 A_{2}}{4}\right)\left\{\frac{15}{16}-\frac{216 \gamma}{16}-\frac{27 \gamma^{2}}{8}-\frac{2225 \epsilon}{192}-\frac{5 \epsilon}{96}-\frac{3260 \gamma \epsilon}{96}+\right.$ $\frac{900 \gamma^{2} \epsilon}{16}+\frac{a^{2 / 3}}{3}\left(\frac{45}{8}-\frac{315 \gamma}{16}-\frac{60 \gamma^{2}}{8}-\frac{525 \epsilon}{192}-\frac{2700 \gamma \epsilon}{96}+\frac{99 \gamma^{2} \epsilon}{16}\right)+$ $\left.\frac{a^{4 / 3}}{3}\left(\frac{90}{8}-\frac{432 \epsilon}{192}-\frac{522 \gamma}{16}-\frac{129 \gamma^{2}}{8}-\frac{2583 \gamma \epsilon}{96}+\frac{99 \gamma^{2} \epsilon}{16}\right)\right\}+\frac{1}{a^{2}}\left(1+3 A_{2}+\right.$ $\left.3 e^{2}\right)$.

Following the method for reducing $\mathrm{H}_{2}$ to the normal form, as in Whittaker (1965), we use the transformation $\mathrm{X}=\mathrm{JT}$ where

$$
X=\left[\begin{array}{c}
x \\
y \\
p_{x} \\
p_{y}
\end{array}\right], T=\left[\begin{array}{l}
Q_{1} \\
Q_{2} \\
P_{1} \\
P_{2}
\end{array}\right]
$$

and $J=\left[J_{i j}\right]_{1 \leq i, j \leq 4}$

$P_{i}=\left(2 I_{i} \omega_{i}\right)^{1 / 2} \operatorname{Cos} \emptyset_{\mathrm{i}}, Q_{i}=\left(\frac{2 I_{i}}{\omega_{i}}\right)^{1 / 2} \operatorname{Sin} \emptyset_{\mathrm{i}}, \mathrm{i}=1,2$.

We follow the approach of Breakwell and Pringle (1966) and choose $\mathrm{J}_{11}=\mathrm{J}_{12}=0, \mathrm{H}_{2}$ takes the form

$H_{2}=\frac{1}{2}\left(P_{1}^{2}-P_{2}^{2}+\omega_{1}^{2} Q_{1}^{2}-\omega_{2}^{2} Q_{2}^{2}\right)$

and

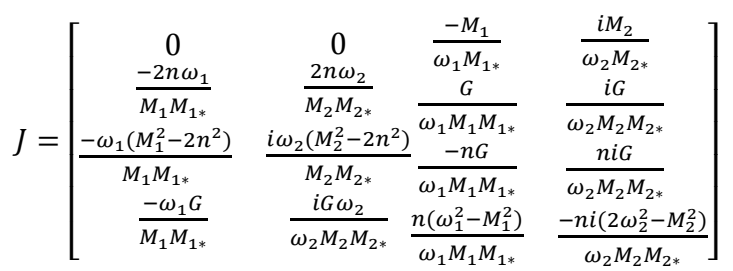

where

$M_{j}=\left(\omega_{j}^{2}-2 F+n^{2}\right)^{1 / 2}$ and $M_{j *}=\sqrt{2}\left(\omega_{j}^{2}-2 E+n^{2}\right)^{1 / 2} \mathrm{j}=1,2$ Applying a contact transformation from $\mathrm{Q}_{1}, \mathrm{Q}_{2}, \mathrm{P}_{1}, \mathrm{P}_{2}$ to $Q_{1}^{\prime \prime}, Q_{2}^{\prime \prime}, P_{1}^{\prime \prime}, P_{2}^{\prime \prime}$ defined by Whittaker (1965)

$P_{j}^{\prime \prime}=\frac{\partial W}{\partial Q_{j}}, Q_{j}^{\prime \prime}=\frac{\partial W}{\partial P_{j}} j=1,2$ and

$W=\sum_{j=1}^{2}\left[Q_{j}^{\prime \prime} \sin ^{-1}\left(\frac{P_{j}}{\sqrt{2 \omega_{\mathrm{j}} \mathrm{Q}_{\mathrm{j}}^{\prime \prime}}}\right)+\frac{\mathrm{P}_{\mathrm{j}}}{2 \omega_{\mathrm{j}}} \sqrt{2 \omega_{\mathrm{j}} \mathrm{Q}_{\mathrm{j}}^{\prime \prime}-\mathrm{P}_{\mathrm{j}}^{2}}\right]$

that is

$Q_{j}=\sqrt{\frac{2 Q_{j}^{\prime \prime}}{\omega_{j}}} \cos P_{j}^{\prime \prime}, P_{j}=\sqrt{2 \omega_{j} Q_{j}^{\prime \prime}} \sin P_{j}^{\prime \prime}, j=1,2$.

Hamiltonian $H_{2}$ is transformed into the form $H_{2}=\omega_{1} Q_{1}^{\prime \prime}-\omega_{2} Q_{2}^{\prime \prime}$. We denote the angular variables $P_{1}^{\prime \prime}$ and $P_{2}^{\prime \prime}$ by $\emptyset_{1}$ and $\emptyset_{2}$ and the actions $Q_{1}^{\prime \prime}$ and $Q_{2}^{\prime \prime}$ by $I_{1}, I_{2}$ respectively. The second order part $\mathrm{H}_{2}$ takes the form $\mathrm{H}_{2}=\omega_{1} \mathrm{I}_{1}-\omega_{2} \mathrm{I}_{2}$. 


\section{Conclusion}

Using Whittaker (1965) method, we have found that the second order part $\mathrm{H}_{2}$ of the Hamiltonian is transformed into the normal form. $H_{2}=\omega_{1} I_{1}-\omega_{2} I_{2}$. We conclude that the values of first and second order components are affected by radiation pressure, oblateness and P-R drag.

\section{Acknowledgements}

We are thankful to D.S.T. Government of India, New Delhi for sanctioning the project SR/S4/MS: 728/11, dated: 15/06/2013 on this topic and IUCAA, Pune for providing local hospitality and facility of library and computer centre for research work.

\section{References}

[1] Arnold,V.I.,(1961).Sovit.Math.Dok1.2,247

[2] Breakwell,j.V.,Pringle,R.,(1966),Celest Mech.\&Dyn.Astro,Vol 17,5574

[3] Bhatnagar,K.B.andHallan,P.P.,(1983),Celest Mech.\& Dyn.Astro.,Vol30,97 .

[4] Chernikov,Yu.A.,(1970).Sov.Astr AJ(14)1,176-181.

[5] Deprit,A.,andDeprit.Bartholome(1967),AJ, Vol-72,173-179.

[6] Ishwar,B.,(1997),CelestMech.\&Dyn.Astro65,253-289. http://dx.doi.org/10.1007/BF00053509.

[7] Kushvah,B.S.,andIshwar,B.,(2006),Journal of dynamical system \& Geometric Theories, Vol- 4(1,79-86).

[8] Liapunov,A.M.(1956),Acad,Sc.USSR.

[9] Murray,C.D.,(1994),Icarus,465-484. http://dx.doi.org/10.1006/icar.1994.1198

[10] Mishra,P.,andIshwar,B.,(1995),Astron J,110, No-4,1901-1904.

[11]Moser.J, (1962), Nach.Akad.Wiss.Gottingen, Math Phys.KI.

[12] Schuerman,D.W.(1980),AstrophysJ,238(1),337-342 http://dx.doi.org/10.1086/157989.

[13] Sahoo, S.K.,Ishwar,B.,(2000),BASI 28,579-586.

[14]Singh,J.,Ishwar,B.,(1999),BASI 27,415

[15] Whittaker (1965), Cambridge University Press, London, 427-430.

[16]Wyatt,S. P.,Whipple,F.L.,(1950),Ap J 111,134- 141. http://dx.doi.org/10.1086/145244 